03

\title{
Физическая модель зависимости числа Нуссельта от числа Рэлея
}

\author{
(С) О.Л. Паточкина, Ю.Г. Казаринов, В.И. Ткаченко \\ Национальный научный центр „Харьковский физико-технический институт“ НАН Украины, \\ 61108 Харьков, Украина \\ ฯ e-mail: tkachenko@kipt.kharkov.ua
}

(Поступило в Редакцию 12 октября 2015 г. В окончательной редакции 12 января 2016 г.)

Физически обоснована и получена в аналитическом виде зависимость числа Нуссельта от числа Рэлея на стадии, предшествующей возникновению турбулентного режима конвекции. На этой стадии число Нуссельта описано степенным законом зависимости от числа Рэлея, содержащим пять констант. Одна константа одинакова для всех жидкостей, и является критическим числом Рэлея $R_{\text {cr }}=1700 \pm 51$. Остальные четыре строго индивидуальны для каждого вида жидкости. Для жидкого гелия критическое число Рэлея $R_{\mathrm{cr}}=35000 \pm 4000$ является пороговым, и равным $\left(R_{1}^{\mathrm{mix}}\right)_{\mathrm{th}}=37085.75$. Оно обусловлено малым отношением диаметра емкости к высоте, а также формированием на дне емкости цилиндрической конвективной ячейки с модовым числом, равным двум, и радиальным числом, определяемым 4-м корнем функции Бесселя первого рода первого порядка.

\section{Введение}

Процессы конвекционного тепломассопереноса могут возникать в ограниченных вязких средах с отрицательным вертикальным градиентом температур. Такие процессы необходимо учитывать при расчетах теплоизоляции трубопроводов и зданий; при построении температурных полей и потоков переноса тепла в отсеках и баках в самолето- и ракетостроении; при анализе теплообмена в замкнутых пространствах начиная от радиоэлектроники, и заканчивая геодезией.

В классической постановке процесс конвекционного тепломассопереноса принято рассматривать на примере задачи переноса теплоты через горизонтальный слой вязкой несжимаемой, подогреваемой снизу жидкости $[1,2]$. При этом практическую значимость приобретают задачи с твердыми границами, когда возмущения скорости на твердых стенках, а также первая производная вертикальной скорости по вертикальной координате равны нулю. Наличие твердых границ позволяет контролировать их температуру, что важно для изучения зависимости параметров конвективного массопереноса от разности температур. К таким параметрам следует отнести числа Нуссельта $(N u)$, Рэлея $(R)$, Прандтля $(P)$ и Грасго́фа (Gr).

Число Нуссельта $(\mathrm{Nu})$ - безразмерное число, определяется отношением полного потока теплоты, определяемого как сумма конвективного $\lambda_{C}$ и за счет теплопроводности $\lambda_{T}$ потока теплоты через слой жидкости к потоку теплоты за счет теплопроводности:

$$
N u=\frac{\lambda_{C}+\lambda_{T}}{\lambda_{T}} .
$$

Число Рэлея $(R)$ - безразмерное число, определяющее поведение вязкой, несжимаемой жидкости под воздействием градиента температуры:

$$
R=\frac{g \beta h^{3}}{v \chi}\left(T_{2}-T_{1}\right),
$$

где $h$ - толщина слоя (расстояние между границами слоя), $g$ - ускорение свободного падения, $\beta$ - коэффициент температурного расширения жидкости, $v-$ кинематическая вязкость жидкости, $\chi-$ коэффициент температуропроводности жидкости, $T_{1}, T_{2}$ - невозмущенная температура верхней и нижней границ слоя соответственно $\left(T_{2}>T_{1}\right)$.

Число Прандтля $(P)$ - безразмерное число, учитывает влияние физических свойств теплоносителя на теплоотдачу:

$$
P=\frac{v}{\chi}
$$

Число Грасго́фа $(G r)$ - безразмерное число, является мерой соотношения архимедовой выталкивающей силы, вызванной неравномерным распределением плотности среды в неоднородном поле температур и вязкими силами:

$$
G r=\frac{R}{P}
$$

Все определяющие физические свойства среды величины в (1)-(4) отнесены к средней температуре в прослойке $0.5\left(T_{1}+T_{2}\right)$. В качестве характерного размера принята толщина слоя $h$. В инженерных расчетах переноса теплоты через емкости и прослойки часто вводится понятие эквивалентного коэффициента теплопроводности [3].

На основании экспериментальных данных в [3,4] показано, что в бесконечном горизонтальном слое, подогреваемом снизу жидкости, конвекция возникает при 
Таблица 1. Режимы конвективных течений в слое вязкой, несжимаемой жидкости в зависимости от числа Рэлея [4]

\begin{tabular}{c|c|c|c}
\hline \multirow{2}{*}{$\begin{array}{c}\text { Номер } \\
\text { области }\end{array}$} & Режим течения жидкости & Диапазон изменения числа Рэлея & Число Нуссельта \\
\hline 1 & Ползучий & $1700<R<3 \cdot 10^{3}$ & $0.0012 R^{0.9}$ \\
\hline 2 & Ламинарный & $3 \cdot 10^{3}<R<2.5 \cdot 10^{4}$ & $0.024 R^{0.25}$ \\
\hline 3 & \multirow{2}{*}{$\begin{array}{c}\text { Трехмерное, переход } \\
\text { в турбулентный }\end{array}$} & $2.5 \cdot 10^{4}<R<3 \cdot 10^{4}$ & $0.3 G r^{0.16} P^{0.21}$ \\
\cline { 1 - 4 } & \multirow{2}{*}{ Турбулентный } & $R>3 \cdot 10^{4}$ & $0.1 G r^{0.31} P^{0.36}$ \\
\hline
\end{tabular}

условии:

$$
R=G r P>R_{\mathrm{cr}},
$$

где $R_{\mathrm{cr}}$ - некоторое критическое значение числа Рэлея, экспериментально установленная величина которого равна $R_{\mathrm{cr}}=1700 \pm 51$.

В результате тщательных экспериментальных исследований выявлены следующие режимы конвективных течений в зависимости от числа Рэлея [4], которые сведены в табл. 1.

Согласно табл. 1, опишем режимы конвективных течений в подогреваемой емкости при условии жестких границ.

В первой области течение в конвективных ячейках может быть названо ползучим вследствие малых скоростей. Жидкость в шестиугольных ячейках поднимается вверх в центре и опускается вниз на их периферии.

При более высоких температурах, т.е. при больших числах Рэлея, наблюдается ламинарной режим конвекции. В этом диапазоне чисел Рэлея при условии существования градиента температур между границами слоя может существовать двумерная структура (в прямоугольной системе координат) течения в виде чередующихся длинных валов, оси симметрии которые параллельны стенкам емкости. При этом по мере увеличения числа Рэлея отношение периода чередующихся восходящих и нисходящих потоков к толщине слоя увеличивается от 2 до 2.8 [4].

При более высоких числах Рэлея течение становится трехмерными, и появляются признаки перехода к турбулентному режиму [4].

Оптические измерения показали, что турбулентный режим в длинном горизонтальном слое жидкости появляется при $R>5 \cdot 10^{4}[4]$.

Детальная информация о пространственной структуре вихревого течения и распределения возмущенной температуры получена благодаря численным решениям уравнений Навье-Стокса [1].

Результаты экспериментальной работы по исследованию числа Нуссельта в обычных вязких, несжимаемых жидкостях [4] дополним экспериментальными данными, полученными для жидкого гелия [5,6]. Эти данные демонстрируют качественное совпадение зависимости числа Нуссельта от числа Рэлея. Однако критические значения числа Рэлея в [1-3] $R_{\mathrm{cr}}=1700 \pm 51$ и в [5] $R_{\text {cr }}=35000 \pm 4000-$ существенно не совпадают. Такое несовпадение авторы объясняют разными значениями отношения диаметра сосуда к высоте, а также теплопроводностью боковой стенки емкости, в которой находился жидкий гелий. В [4] отношение диаметра емкости к высоте достаточно велико - $\Gamma=16$, в то время как в работе [6] оно равнялось $\Gamma=0.5$. Учет теплопроводности боковой стенки позволил расширить диапазон критического числа Рэлея до $2 \cdot 10^{4}<R_{\mathrm{cr}}<6 \cdot 10^{4}[6]$, что, однако, не изменило абсолютную величину критического числа Рэлея. Поэтому остается открытым вопрос о причине большого значения критического числа Рэлея, обнаруженного в серии экспериментов в жидком гелии.

В последнее время в работах $[7,8]$ предложено рассматривать возникновение ячеистых структур, в том числе ячеек Бенара, как результат появления и последующей плотной упаковки большого количества элементарных цилиндрических конвективных ячеек, диаметр которых определяется из вида граничных условий. В цитированных работах в результате решения линейного стационарного уравнения Навье-Стокса в цилиндрической геометрии при наличии твердых границ получены аналитические выражения для возмущенных скорости и температуры в цилиндрической конвективной ячейке. Теоретически показано, что диаметр цилиндрической конвективной ячейки с твердыми границами обратно пропорционален значению минимального волнового числа $\left(k_{r}\right)_{\text {rigid }}=3.116 h^{-1}$ и составляет величину $2.45 h$.

Введение понятия элементарной цилиндрической конвективной ячейки в задачах о конвективных течениях в слоях вязких, несжимаемых жидкостей позволяет по-новому рассмотреть процесс формирования режимов конвективных течений.

Поэтому в настоящей работе на основе полученных ранее другими авторами экспериментальных данных и предложенной авторами теоретической модели элементарной конвективной ячейки дано физическое обоснование возникновения ползучего режима конвективного течения и степенной зависимости числа Нуссельта от числа Рэлея на начальных стадиях возникновения конвекции. В работе также дано обоснование большого значения критического числа Рэлея в жидком гелии. 


\section{1. Теоретическая модель описания тепловой конвекции и определение диаметра элементарной конвективной ячейки}

Линеаризованные уравнения Навье-Стокса и теплопроводности [1] в слое с плоскими границами для цилиндрической конвективной ячейки описывают возмущенные значения вертикальной скорости $v_{z}$ и температуры $T$ :

$$
\begin{gathered}
\frac{\partial}{\partial t} \Delta v_{z}=\Delta \Delta v_{z}+R \Delta_{\perp} T, \\
P \frac{\partial T}{\partial t}=\Delta T+v_{z},
\end{gathered}
$$

где $\Delta=\Delta_{\perp}+\frac{\partial^{2}}{\partial z^{2}}-$ оператор Лапласа, $\Delta_{\perp}=\frac{1}{r} \frac{\partial}{\partial r}\left(r \frac{\partial}{\partial r}\right)-$ поперечный лапласиан, в котором на основании аксиальной симметрии ячеек отсутствует слагаемое, характеризующее зависимость возмущений от азимутального угла, т. е. полагаем $\partial \ldots / \partial \phi=0$.

При приведении системы уравнений (6), (7) к безразмерному виду использованы следующие характерные единицы измерения: единица длины - толщина слоя $h$, единица времени - $\tau=h^{2} v^{-1}$, единица температуры $\Theta$, координата $z$ обезразмерена на толщину слоя и изменяется в интервале $0 \leq z \leq 1$.

Система уравнений должна быть дополнена граничными условиями. В рассматриваемом случае полагаем границы слоя твердыми, что налагает на решения системы уравнений (6), (7) следующие условия на границах $z=0$ и $z=1$ слоя $[1,4]$ :

$$
v_{r}=v_{z}=0 ; \quad T=0 ; \quad \frac{d v_{z}}{d z}=0 .
$$

Уравнения (6), (7) имеют частные решения, которые описывают временную динамику возмущений вертикальной скорости и температуры в цилиндрической ячейке [8]:

$$
\begin{gathered}
v_{z}(r, z, t)=v(z) J_{0}\left(k_{r} r\right) \exp (-\lambda t), \\
T(r, z, t)=\vartheta(z) J_{0}\left(k_{r} r\right) \exp (-\lambda t),
\end{gathered}
$$

где $\lambda-$ собственные числа, характеризующие затухание $(\lambda>0)$, нарастание $(\lambda<0)$ или стационарное состояние $(\lambda=0)$ возмущений $(9),(10) ; v(z)$ и $\vartheta(z)$ - амплитуды возмущений вертикальной скорости и температуры соответственно; $J_{0}(x)$ - функции Бесселя первого рода нулевого порядка от аргумента $x ; k_{r}$ - радиальное волновое число, характеризующее зависимость возмущений от поперечной координаты $r$.

На практике наибольший интерес представляют стационарные решения системы уравнений (9), (10), так как они наиболее точно описывают экспериментально наблюдаемое явление длительного существования пространственно-периодических конвективных структур.
Амплитуды стационарных $(\lambda=0)$ возмущений в выражениях (9), (10) имеют вид:

$$
\begin{gathered}
v(z)=A_{1}\left(1-\operatorname{ch}\left[\left(z-\frac{1}{2}\right) X_{+}\right] \operatorname{ch}^{-1}\left(\frac{X_{+}}{2}\right)\right) \sin (z \sqrt{z-b}), \\
\vartheta(z)=-\frac{1}{\sqrt{b}} \int_{0} v(\xi) \operatorname{sh}(\sqrt{b}(\xi-z)) d \xi \\
\left.+\frac{1}{\sqrt{b}} \frac{\operatorname{sh}(z \sqrt{b})}{\operatorname{sh}(\sqrt{b})} \int_{0}^{1} v(\xi) \operatorname{sh}(\sqrt{b}(\xi-1)) d \xi, \quad 12\right)
\end{gathered}
$$

где

$$
\begin{gathered}
a=\left(k_{r}^{2} R\right)^{\frac{1}{2}}, \quad b=k_{r}^{2} \\
X_{ \pm}=\left(0.5\left(\sqrt{(0.5 a+b)^{2}+0.75(a)^{2}} \pm(0.5 a+b)\right)\right)^{\frac{1}{2}} .
\end{gathered}
$$

Исходя из вида решений (9), (10) и основываясь на методике определения диаметра конвективной ячейки [8], можно определить характерный диаметр элементарной конвективной ячейки с твердыми границами. Его теоретическое значение равно

$$
\begin{aligned}
\left(D_{c}\right)_{\text {rigid }} & =2\left(R_{c}\right)_{\text {rigid }}=2 \sigma_{1, i} /\left(k_{r}\right)_{\text {rigid }} \\
& \approx 2 \cdot 3.832 / 3.116 \approx 2.46,
\end{aligned}
$$

где $R_{c}-$ радиус конвективной ячейки, $\sigma_{1, i}-$ $i$-й нуль функции Бесселя первого рода первого порядка $\left(J_{1}\left(\sigma_{1, i}=0\right)\right), i=1,2,3, \ldots$ Приведем значения первых пяти нулей функции Бесселя: $\sigma_{1,2}=3.832, \sigma_{1,2}=7.016$, $\sigma_{1,3}=10.173, \sigma_{1,4}=13.324, \sigma_{1,5}=16.471$.

Экспериментально измеренная величина диаметра конвективной ячейки по данным работы [4] оказывается равной 2.31. По данным работы [8] его величина варьируется в диапазоне $2.2-2.4$.

Таким образом, теоретически определенное значение диаметра конвективной ячейки с твердыми границами в единицах толщины слоя определяется величиной 2.46 и с учетом погрешностей измерения соответствует экспериментально измеренному значению.

\section{2. Физическое обоснование степенной зависимости числа Нуссельта от числа Рэлея на основе модели элементарной конвективной ячейки}

Из основательных экспериментальных исследований зависимости числа $N u$ от числа $R$ для различных вязких жидкостей (вода, силиконовое масло АК 3 и АК 350, этиленгликоль, гептан) [4] следует, что для чисел Рэлея $R \geq R_{\text {сr }}$ и вплоть до $R \leq 3 \cdot 10^{3}$ число $N u$ зависит от числа $R$ по степенному закону, где степень близка к единице. Далее в интервале $3 \cdot 10^{3}<R<2.5 \cdot 10^{4}$ степень уменьшается до 0.25 . С дальнейшим увеличением числа Рэлея начинает проявляться зависимость числа Прандтля от температуры, и число $N u$ уже не является функцией только числа $R$. 
Таблица 2. Значения констант для вычисления числа Нуссельта по формуле (17)

\begin{tabular}{c|c|c|c|c|c|c}
\hline Константа & Вода & $\begin{array}{c}\text { Силиконовое } \\
\text { масло АК 3 }\end{array}$ & $\begin{array}{c}\text { Силиконовое } \\
\text { масло АК 350 }\end{array}$ & Этилен-гликоль & Гептан & Жидкий гелий \\
\hline$R_{\text {cr }}$ & 1700 & 1700 & 1700 & 1700 & 1700 & 37085 \\
$\alpha$ & 6.91106 & 5.24407 & 2.49929 & 2.55875 & 1.80979 & 1.10213 \\
$\gamma$ & 1.16866 & 1.04303 & 0.47338 & 0.44065 & 0.20957 & 0.43462 \\
$\delta$ & 7.81554 & 5.8945 & 2.59367 & 2.51563 & 1.80836 & 0.209907 \\
$\mu$ & 1.90041 & 1.72707 & 1.14322 & 1.16526 & 1.02159 & 1.18853
\end{tabular}

Дадим физическое объяснение явления степенной зависимости числа $N u$, определяемого выражением (1), от числа $R$ при сравнительно небольших числах $R$.

Количество переносимого за счет конвекции тепла $\lambda_{C}$ определяется через плотность теплового потока $\bar{\lambda}_{C}$ выражением $\lambda_{C}=S_{1} \bar{\lambda}_{C}$, где $S_{1}-$ площадь, занимаемая одной или ансамблем элементарных конвективных ячеек. Эта площадь не может превышать площади жидкости $S_{0}$, которую она занимает в рассматриваемой емкости $S_{1} \leq S_{0}$.

В отсутствие конвективных ячеек поток переносимой за счет тепловой диффузии теплоты $\lambda_{T}$ определяется через плотность диффузионного потока $\bar{\lambda}_{T}$ выражением $\lambda_{T}=S_{0} \bar{\lambda}_{T}$. С появлением конвективных ячеек полное количество переносимой теплоты $\lambda_{C}+\lambda_{T}$ определяется суммой диффузионного потока через площадь, свободную от конвективных ячеек $\left(S_{0}-S_{1}\right)$, и конвективного потока, переносимого конвективными ячейками через площадь $S_{1}$, т. е. для полного количества переносимой теплоты за счет тепловой диффузии и конвекции справедливо выражение

$$
\lambda_{C}+\lambda_{T}=\left(S_{0}-S_{1}\right) \bar{\lambda}_{T}+S_{1} \bar{\lambda}_{C},
$$

где $\bar{\lambda}_{C}$ - плотность конвективного потока теплоты.

Учитывая вышеизложенное, выражение (1) можно представить в виде

$$
N u=1+\frac{S_{1}}{S_{0}}\left(\frac{\bar{\lambda}_{C}}{\bar{\lambda}_{T}}-1\right),
$$

где $\bar{\lambda}_{C} \geq \bar{\lambda}_{T}$.

Согласно закону Ньютона-Рихмана, плотность теплового потока пропорциональна температурному напору (разности температур $\left(T_{2}-T_{1}\right)$ ) [9,10]. Ввиду этого выражение в скобках в (14) в результате последовательных преобразований может быть представлено в виде

$$
\begin{aligned}
\frac{\bar{\lambda}_{C}}{\bar{\lambda}_{T}}-1 & =\frac{\xi_{C}\left(T_{2}-T_{1}\right)}{\bar{\lambda}_{T}}-1=\frac{\xi_{C}\left(T_{2}-T_{1}\right)}{\bar{\lambda}_{T} \frac{g \beta h^{3}}{v \chi}} \frac{g \beta h^{3}}{v \chi}-1 \\
& =\frac{R}{\bar{\lambda}_{T} \frac{g \beta h^{3}}{v \chi \xi_{C}}}-1=\frac{R}{R_{\mathrm{cr}}}-1,
\end{aligned}
$$

где $\xi_{C}-$ коэффициент пропорциональности.

В (15) принято $\bar{\lambda}_{T} \frac{g \beta h^{3}}{v \chi \xi_{C}}=R_{\mathrm{cr}}$, так как вблизи критической точки при $R \geq R_{\mathrm{rc}}$, согласно экспериментальных данных [4], число Нуссельта изменяется по закону $N u \simeq 1+\left(R R_{\mathrm{cr}}^{-1}-1\right)^{W}$, где показатель степени $W$ больше единицы.

Поскольку появление конвективных ячеек происходит при $R>R_{\text {cr }}$, то площадь $S_{1}$, которую они образуют в слое жидкости верхними границами, также может быть описана величиной, пропорциональной превышению числа Рэлея над его критическим значением в некоторой степени $\gamma: S_{1} S_{0}^{-1}=\alpha\left(R R_{\mathrm{cr}}^{-1}-1\right)^{\gamma}$, где $\alpha-$ коэффициент пропорциональности.

Таким образом, учитывая экспериментально полученные зависимости числа Нуссельта от числа Рэлея $R$ для различных жидкостей [4], где показатель степени $\left(R R_{\mathrm{cr}}^{-1}-1\right)$ уменьшается от величины, большей единицы $(\gamma+1)$ (при $\left.1700<R<3 \cdot 10^{3}\right)$ до некой величины $\mu$ $(\mu<\gamma)$ (при $\left.R>3 \cdot 10^{3}\right)$, окончательно запишем его в виде

$$
N u=1+\alpha\left(R R_{\mathrm{cr}}^{-1}-1\right)^{\gamma+1}\left(1+\delta\left(R R_{\mathrm{cr}}^{-1}-1\right)^{\mu}\right)^{-1},
$$

где константы $\alpha, \delta, \gamma, \mu$ определяются данными экспериментов [4].

\section{1. Вязкие несжимаемые жидкости в емкостях с большим отношением диаметра емкости к высоте}

Ниже на рисунке приведены результаты сопоставления теоретической зависимости (16) (сплошная линия) с экспериментальными данными (изображены точками) работы [4] для различных видов вязких, несжимаемых жидкостей. В табл. 2 даны значения подгоночных констант $\alpha, \delta, \gamma, \mu$ в выражении (16).

Из рисунка и табл. 2 следует, что полученная аналитическая зависимость характеризуется достаточно высокой точностью описания экспериментальных данных. Это указывает на применимость предложенной концепции элементарной конвективной ячейки для обоснования физической сущности числа Нуссельта.

Таким образом, в настоящем разделе для вязких несжимаемых жидкостей в емкостях с большим отношением диаметра емкости к высоте в режимах ползучего и ламинарного конвективных течений получены аналитические зависимости числа Нуссельта от числа Рэлея. Эти зависимости характеризуются индивидуальным набором констант для каждого вида жидкости. 

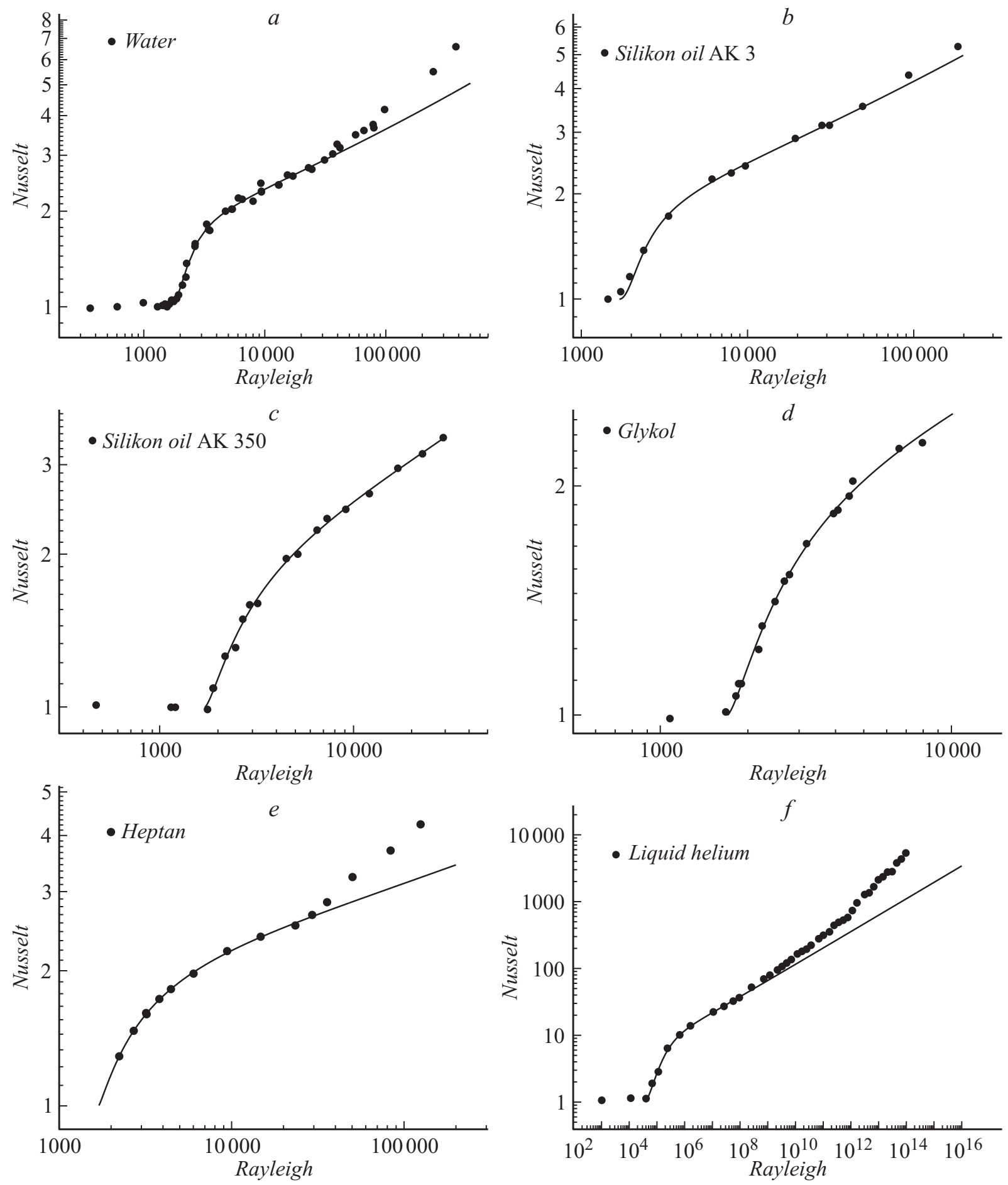

Зависимость числа Нуссельта от числа Рэлея для различных жидких сред.

Одинаковым для всех жидкостей является критическое число Рэлея: $R_{\mathrm{cr}}=1700 \pm 51$. После точки перегиба (область перехода конвективных течений в турбулентный режим, где $R>5 \cdot 10^{4}$ ) использование функциональной зависимости (16) неправомерно. Для чисел Рэлея в интервале $R>5 \cdot 10^{4}$ реализуется турбулентный режим течения, который характеризуется отмеченным в [4] слабым степенным ростом числа $\mathrm{Nu}$ от числа $R$.

\section{2. Вязкие несжимаемые жидкости в емкостях с малым отношением диаметра емкости к высоте. Жидкий гелий}

Отдельно рассмотрим экспериментальные данные, полученные в работе [6] для жидкого гелия. На рисунке, $e$ приведена зависимость числа Нуссельта от числа Рэлея для жидкого гелия. Для аналитического описания этой 
зависимости применимо выражение (16), где вместо критического числа Рэлея $R_{\text {cr }}$ должно быть использовано пороговое $R_{\text {th }}$. Такую замену можно объяснить следующим образом.

При больших отношениях диаметра емкости к высоте в слое жидкости с ростом числа Рэлея ячейки располагаются рядом друг с другом и их радиус определяется толщиной слоя жидкости [7]. Число Нуссельта при больших отношениях диаметра емкости к высоте определяется, как это следует из предыдущего раздела, критическим числом Рэлея $R_{\mathrm{cr}}$.

При малых отношениях диаметра сосуда к высоте радиус конвективных ячеек определяется радиусом сосуда [11]. С увеличением числа Рэлея конвективные ячейки будут появляться последовательно одна над другой снизу вверх, заменяя по всему объему емкости термодиффузионный теплообмен горизонтальными слоями конвективных ячеек. Поэтому при заданном радиусе емкости число Нуссельта будет определяться не критическим числом Рэлея $R_{\mathrm{cr}}$, а пороговым $-R_{\mathrm{th}}$, которое принадлежит нейтральной кривой [1].

Большое значение порогового (критического) числа Рэлея для жидкого гелия $R_{\mathrm{cr}}=35000 \pm 4000$ [6], на наш взгляд, может объясняться тем, что модовое число $n$ аналитического решения задачи Рэлея с твердыми границами [8] должно равняться 2. Такой вывод основан на том, что только при $n=2$ достигаются числа Рэлея, близкие к $R_{\mathrm{cr}}$.

При определении модового числа ход рассуждений следующий.

Из аналитического решения задачи Рэлея с твердыми границами можно построить аналитическое решение со смешанными границами, когда нижняя граница твердая, а верхняя - свободная (метод изложен в [1]). Соответствующая зависимость числа Рэлея от волнового числа (нейтральная кривая) для смешанных границ может быть получена из анализа свойств цилиндрической конвективной ячейки с твердыми границами, где число Рэлея определено в виде $R=a^{3} / b[8]$ (см. также (12)). Из этого выражения, а также из аппроксимации табличных данных [1] можно получить приближенное аналитическое выражение для числа Рэлея с твердыми границами, а затем отмеченным выше методом и для смешанных границ.

В результате выполнения перехода от твердых границ к смешанным зависимость числа Рэлея от волнового числа можно записать в виде

$$
R_{1}^{\operatorname{mix}}\left(k_{r}\right) \simeq \alpha_{2} \frac{\left(2^{2} \pi^{2}+\left(\beta_{2} k_{r}\right)^{\mu_{2}}\right)^{3}}{2^{4}\left(\beta_{2} k_{r}\right)^{\mu_{2}}},
$$

где $\alpha_{2}=1.674, \beta_{2}=0.814, \mu_{2}=2.04$.

Как сказано ранее, большое значение порогового числа Рэлея наиболее соответствует, на наш взгляд, конфигурации конвективных валов, состоящей из четырех концентрических валов, аналог которых описан в [2]. Под- ставляя в (17) волновое число $k_{r}=\sigma_{1,4} / R_{c}=2 \sigma_{1,4} / 2 \Gamma$ $=2 \sigma_{1,4}$, где $\sigma_{1,4}=13.324-4$-й нуль функции Бесселя первого рода первого порядка $\left(J_{1}\left(\sigma_{1,3}\right)=0\right)[11], R_{c}-$ радиус ячейки, определим значение порогового числа Рэлея $\left(R_{1}^{\mathrm{mix}}\right)_{\mathrm{th}}=37085.75$ и соответствующее ему значение волнового числа $\left(k_{r}\right)_{\mathrm{th}}=26.648$.

Как видно из приведенного аналитического расчета, для жидкого гелия пороговое число Рэлея $R_{\text {th }}$ достаточно близко (в рамках указанной экспериментальной погрешности) расположено к найденному экспериментально.

Таким образом, экспериментально обнаруженное большое пороговое число Рэлея для жидкого гелия обусловлено малым отношением диаметра емкости к высоте (как это справедливо отмечено в [6]), и может быть объяснено моделью элементарной конвективной ячейки со смешанными граничными условиями при большом количестве концентрических конвективных валов в горизонтальной плоскости.

\section{Выводы}

Рассмотрены решения линейной задачи Рэлея с твердыми и смешанными граничными условиями. Показано существование в конвективном слое цилиндрической элементарной конвективной ячейки.

На основе полученных ранее другими авторами экспериментальных данных и предложенной авторами теоретической модели цилиндрической элементарной конвективной ячейки дано физическое обоснование возникновения ползучего режима конвективного течения и степенной зависимости числа Нуссельта от числа Рэлея на начальных стадиях возникновения конвекции в емкостях с большим отношением диаметра емкости к высоте.

На основе теоретических расчетов и экспериментальных данных показано, что диаметр элементарной цилиндрической конвективной ячейки с твердыми граничными условиями в единицах толщины слоя определяется величиной 2.46.

Для вязких несжимаемых жидкостей в емкостях с большим отношением диаметра емкости к высоте в режимах ползучего и ламинарного конвективных течений физически обоснована и получена аналитическая зависимость числа Нуссельта от числа Рэлея. Эта зависимость характеризуется индивидуальным набором констант для каждого вида жидкости. Одинаковым для всех жидкостей является критическое число Рэлея: $R_{\mathrm{cr}}=1700 \pm 51$.

Показано, что после точки перегиба (область перехода конвективных течений в турбулентный режим) использование полученной аналитической кривой неправомерно. Для чисел Рэлея в этом интервале и выше реализуется турбулентный режим течения, который характеризуется слабым степенным ростом числа Нуссельта от числа Рэлея. 
Показано, что при заданном радиусе емкости, когда отношение диаметра емкости к высоте невелико, аналитическое выражение для числа Нуссельта будет определяться не критическим числом Рэлея $R_{\mathrm{cr}}$, а пороговым $-R_{\mathrm{th}}$, которое принадлежит нейтральной кривой. Таким образом, экспериментально обнаруженное большое пороговое число Рэлея для жидкого гелия обусловлено малым отношением диаметра емкости к высоте и может быть объяснено моделью элементарной конвективной ячейки со смешанными граничными условиями при большом количестве концентрических конвективных валов в горизонтальной плоскости.

\section{Список литературы}

[1] Гершуни Г.З., Жуховицкий Е.M. Конвективная устойчивость несжимаемой жидкости. М: Наука, 1972. С. 393.

[2] Koschmieder E.L. Bénard Cells and Taylor Vortices. Cambridge University Press. 1993. Р. 350.

[3] Исаев С.И., Кожинов И.А., Кофанов В.И. и др. // Теория тепломассобмена. М.: Высшая школа, 1979. С. 341-345.

[4] Silveston P.L. Wärmedurchgang in waagerechten Flüssigkeitsschichten. Forsch. Ing. Wes. 1958. Bd 24. 29. P. 59-69.

[5] Manneville P. Rayleigh-Benard convection: Thirty years of experimental. Theoretical and modeling work. Springer Tracts in Modern Physics, 2006. Vol. 207. P. 41-60.

[6] Chavanne X., Chilla F., Chabaud B., Castaing B., Chaussy J., Herbral B. // J. Low Tem. Phys. 1996. Vol. 104. N 1/2. P. $109-129$.

[7] Bozbiei L.S., Borts B.V., Kostikov A.O., Tkachenko V.I. // E. Eur. J. Phys. For. 2014. Vol. 1. N 4. P. 49-56.

[8] Patochkina O.L., Borts B.V., Tkachenko V.I. // E. Eur. J. Phys. 2015. Vol. 2. N 1. P. 23-32.

[9] Ньютон И. Математические начала натуральной философии. Оптика. Оптические лекции. Л.: Изд-во П.П. Сойкин, 1929. C. 72.

[10] Рихман В. Труды по физике. М.: Изд-во АН СССР, 1956. C. 711.

[11] Bozbiei L., Borts B., Kazarinov Yu., Tkachenko V. // Energetika. 2015. T. 61. N 2. P. 45-56. 